\title{
Angle-tunable intersubband photoabsorption and enhanced photobleaching in twisted bilayer graphene
}

\author{
Eva A. A. Pogna ${ }^{1}$, Xianchong Miao $^{2}$, Driele von Dreifus $^{3}$, Thonimar V. Alencar ${ }^{4}$, Marcus V. O. Moutinho ${ }^{5}$,

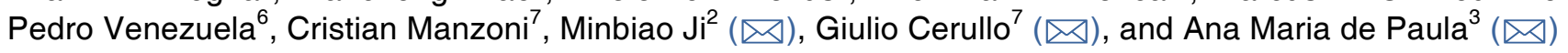 \\ ${ }^{1}$ Istituto di Nanoscienze CNR-NANO, Laboratory NEST, Piazza San Silvestro 12, Pisa 56127, Italy \\ ${ }^{2}$ State Key Laboratory of Surface Physics and Department of Physics, Fudan University, Shanghai 200433, China \\ ${ }^{3}$ Departamento de Física, Instituto de Ciências Exatas, Universidade Federal de Minas Gerais, Belo Horizonte-MG 31270-901, Brazil \\ ${ }^{4}$ Departamento de Física, Instituto de Ciências Exatas e Biológicas, Universidade Federal de Ouro Preto, Ouro Preto-MG 35400-000, Brazil \\ ${ }^{5}$ Núcleo Multidisciplinar de Pesquisas em Computação - NUMPEX-COMP, Campus Duque de Caxias, Universidade Federal do Rio de Janeiro, \\ Duque de Caxias-RJ 25265-970, Brazil \\ ${ }^{6}$ Instituto de Física, Universidade Federal Fluminense, UFF, Niterói-RJ 24210-346, Brazil \\ ${ }^{7}$ IFN-CNR, Dipartimento di Fisica, Politecnico di Milano, Piazza L. da Vinci 32, Milano 20133, Italy
}

(C) The Author(s) 2021

Received: 15 July 2020 / Revised: 6 December 2020 / Accepted: 7 December 2020

\begin{abstract}
Van der Waals heterostructures obtained by artificially stacking two-dimensional crystals represent the frontier of material engineering, demonstrating properties superior to those of the starting materials. Fine control of the interlayer twist angle has opened new possibilities for tailoring the optoelectronic properties of these heterostructures. Twisted bilayer graphene with a strong interlayer coupling is a prototype of twisted heterostructure inheriting the intriguing electronic properties of graphene. Understanding the effects of the twist angle on its out-of-equilibrium optical properties is crucial for devising optoelectronic applications. With this aim, we here combine excitation-resolved hot photoluminescence with femtosecond transient absorption microscopy. The hot charge carrier distribution induced by photo-excitation results in peaked absorption bleaching and photo-induced absorption bands, both with pronounced twist angle dependence. Theoretical simulations of the electronic band structure and of the joint density of states enable to assign these bands to the blocking of interband transitions at the van Hove singularities and to photo-activated intersubband transitions. The tens of picoseconds relaxation dynamics of the observed bands is attributed to the angle-dependence of electron and phonon heat capacities of twisted bilayer graphene.
\end{abstract}

\section{KEYWORDS}

twisted bilayer graphene, transient absorption microscopy, hot-photoluminescence, van der Waals heterostructures, optoelectronic properties, van Hove singularities

\section{Introduction}

Van der Waals heterostructures composed of stacked singlelayer crystals represent a promising platform for novel electronic, photonic and spintronic devices [1-8]. Wide tunability of the optoelectronic properties is achieved by both selection of the stacked materials and control of the relative rotation between the crystal registries $[5,9,10-12]$, i.e. the twist angle. In the case of two layers of graphene, strong interlayer coupling drives the hybridization of the electronic states and can lead to the formation of moire super-lattices for finite twist angle. The rotational misalignment of twisted bilayer graphene (tBLG) enables to explore also new properties as topological valley transport [13], superconductivity [14] at the magic twist angle $\theta \sim 1.1^{\circ}$, or the topologically protected one-dimensional (1D) chiral states appearing at the moiré domain walls [15]. The Dirac cones associated to the two layers in $\mathrm{BLLG}$ are no longer aligned in the energy-momentum landscape and two saddle points appear at their low-energy intersections. The saddle points contribute to van Hove singularities (vHs) in the density of states, which occur at energies $E_{\mathrm{vHs}}$ that depend on the twist angle [16]. The vHs are detected as a peak in the optical conductivity of tBLG [17-19] and they have been related to the increase of hot photoluminescence (PL) for resonant excitation [20]. These vHs appear at much lower energies than in single-layer graphene (SLG) [16, 21-24] and allow angle controlled visible and near-infrared (NIR) absorption enhancement. This property has been exploited in a tBLG based photo-detector to selectively increase the photocurrent generation at the vHs [25].

The changes in crystal symmetry, electronic band structure and optical conductivity come along with modifications of the relaxation pathways available to charge carriers driven out of equilibrium. The carrier dynamics in SLG is well-known as it has been intensively studied by means of ultrafast transient absorption (TA) [26-31], time-resolved photocurrent $[32,33]$ and photoemission spectroscopies [34-36]. Excitation with femtosecond light pulses produces out-of-equilibrium distributions of electrons and holes in the conduction and valence bands, which thermalize in $\sim 50 \mathrm{fs}$ via Coulomb 
two-body scattering to hot Fermi-Dirac (FD) distributions $[30,31]$. The relaxation of the hot carriers proceeds through scattering with strongly coupled optical phonons (SCOP) till a common temperature is reached in about $200 \mathrm{fs}$. The cooling of the thermalized electron-phonon bath is finally driven by the lattice and achieved in less than 10 picoseconds via anharmonic decay of the hot phonons. In defected SLG, hot carriers can release their excess energy also by defect-mediated emission of acoustic phonons (supercollision mechanism), which occurs on a picosecond time scale dependent on substrate and defect densities [31, 33, 37-43]. The hot carrier distribution inhibits interband absorption over a broad energy range due to Pauli blocking, so that in the TA spectra of SLG a decreased absorption, or photo-bleaching (PB), signal is detected.

In Bernal stacked bilayer graphene, together with the $\mathrm{PB}$ band, Limmer et al. [44] observed a photoinduced absorption (PA) with a single decay time of $5 \mathrm{ps}$, about twice as long as the PB signal. The PA has been attributed to transitions between subbands formed within the conduction and the valence bands, named intersubband (ISB) transitions, which are absent in SLG since it lacks a subband structure. Also time- and angle-resolved photoemission spectroscopy (tARPES) has demonstrated long-living (up to $10 \mathrm{ps}$ ) population inversion in bilayer graphene $[45,46]$ attributed to the presence of a small gap. Previous studies in tBLG by TA microscopy [47, 48] and tARPES [49] have explored the twist-angle dependence and shown long decay times attributed to bound excitons and to states reached by two-photon absorption $[48,50]$.

Here we studied the out-of-equilibrium optical properties of tBLG as a function of the twist angle by femtosecond TA microscopy with high sensitivity, which allows to explore low excitation regimes in which the angle-dependent contribution from ISB transitions emerges. Specifically, we investigated heterostructures with the vHs located in the NIR and twist angle $\theta$ in the range of $\sim 6^{\circ}-8^{\circ}$. The tBLG samples were prepared by chemical vapor deposition, as described in the Methods, and have tens of $\mu \mathrm{m}$ linear dimension. A lattice rotation in the real space by an angle $\theta$ corresponds to a rotation of $\theta$ in the phase-space of the hexagonal Brillouin zones (BZs), as sketched in Figs. 1(a) and 1(b). Accordingly, the Dirac points of the two layers (see Fig. $1(\mathrm{c})$ ), $\mathrm{K}_{\mathrm{A}}$ and $\mathrm{K}_{\mathrm{B}}$, no longer coincide and are displaced by a momentum $\Delta K=2 K \sin (\theta / 2)$, where $K=$ $4 \pi / 3 a$ and $a \approx 0.246 \mathrm{~nm}$ are the SLG lattice parameter [51]. The interlayer interactions perturb the Dirac cones of each layer causing the formation of minigaps with the same energy in the conduction and valence bands at the vHs, as is schematically shown in Fig. 1(c).

Figure 1(d) shows the optical microscope image of one of the investigated samples, named stack 1 , measured in reflection. It consists of a large single-crystal SLG, with an inner brighter region, where a second graphene layer is stacked to form a bilayer single crystal. A first determination of the mutual orientation of the lattices in the bilayer domain is obtained by extrapolation of the hexagonal habits of the outer/inner growth regions, corresponding respectively to the 1 st/2nd layer [52]. In stack 1 , the hexagons are rotated by an angle $\theta=7^{\circ} \pm 1^{\circ}$. The angle determination from the optical images is affected by a large uncertainty related to the definition of the single-crystals shape. Twist angle is usually measured by high-resolution transmission electron microscopy [53,54], scanning tunneling microscopy [16] and lattice resolution atomic force microscopy [55]. Here we adopted an all-optical, far-field approach, based on measuring the hot-photoluminescence excitation (hot-PLE) spectrum under femtosecond laser excitation. As previously observed [20], due to the enhancement of pump absorption, a peak is expected in the bilayer emission when the laser is tuned at the vHs. In Fig. 1(e) the hot PL intensity of tBLG normalized to that of SLG $\left(I_{\mathrm{BBLG}} / I_{\mathrm{SLG}}\right)$ is shown as function of laser excitation energy. The experimental data are fitted with a Gaussian function centered at $E_{\mathrm{vHs}}=1.388 \pm 0.007 \mathrm{eV}$. From the energy of the vHs, we extracted a more accurate estimation of the twist angle considering the correlation $\theta\left(^{\circ}\right)=A-\sqrt{\left(B-C \cdot E_{\mathrm{vHs}}(\mathrm{eV})\right)}$, with $A=45.380, B=2.030 \times 10^{3}, C=4.186 \times 10^{2}$ extracted from the analysis of the calculated joint density of states (JDOS) as a function of $\theta$ reported in Fig. S1 in the Electronic Supplementary Material (ESM). We obtained the value $\theta=$ $7.31^{\circ} \pm 0.04^{\circ}$ for stack 1 , in good agreement with the evaluation from the optical image inspection. The hot PL map in Fig. 1(e) (a)

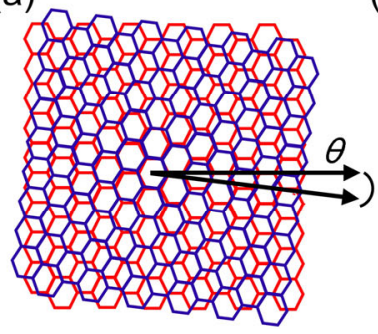

(b)

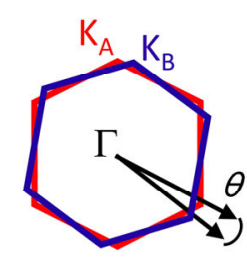

(c)

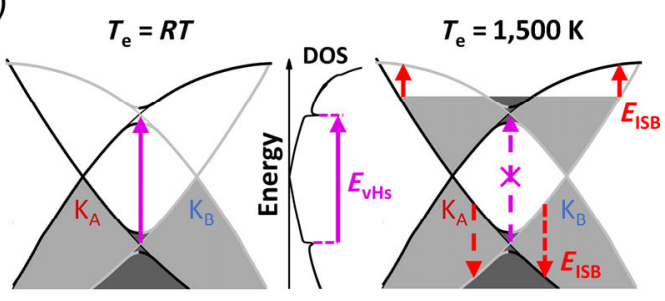

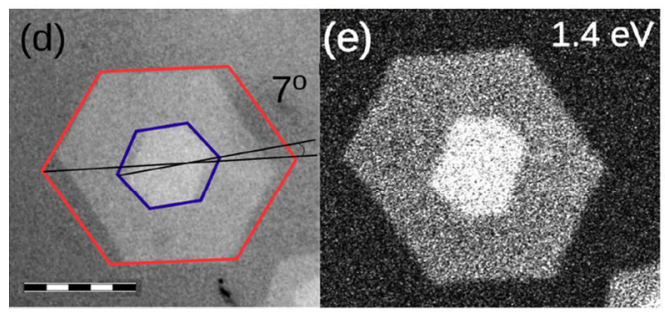
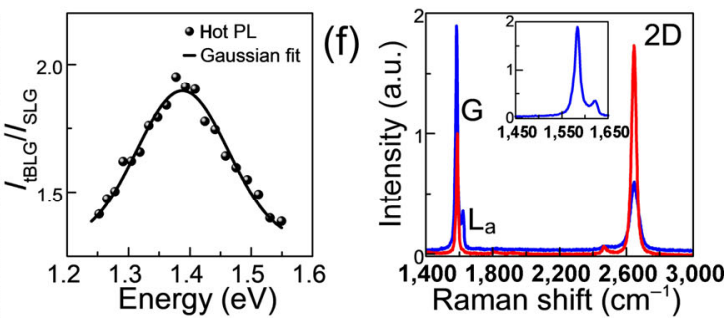

Figure 1 (a) Sketch of the crystal structure of a tBLG with twist angle $\theta$; (b) the corresponding BZs of two rotated graphene layers as the red and blue hexagons; (c) energy structure indicating the optical transition at the $\mathrm{vHs}$ at photon energy $E_{\mathrm{vHs}}$ (magenta line) and intersubband transitions from photo-excited FD distributions of electrons (red solid lines) and holes (red dashed lines); (d) bright field optical image of stack 1 with blue and red hexagons identifying a layer rotation angle of about $7^{\circ}$, scale bar $10 \mu \mathrm{m}$; (e) PL intensity map for laser excitation energy of $1.4 \mathrm{eV}$, in resonance with the $E_{\mathrm{vHs}}$ identified by the peak in the PLE intensity of $\operatorname{tBLG}\left(I_{\mathrm{tBLG}}\right)$ divided by that of the corresponding SLG $\left(I_{\mathrm{SLG}}\right)$; (f) Raman spectra of stack 1 at the bilayer (blue line) and monolayer (red line) regions normalized by the intensity of the $\mathrm{G}$ peak. The inset shows a zoom-in of the bilayer $\mathrm{G}$ and $\mathrm{L}_{\mathrm{a}}$ peaks divided by the monolayer G peak. 
for laser excitation tuned at $1.4 \mathrm{eV}$, close to $E_{\mathrm{vHs}}$, reports indeed $a \approx 2$ times higher emission intensity at the tBLG compared to the SLG, uniform across the bilayer domain.

Extra peaks have been observed in visible, IR and UV Raman spectra of tBLG compared to SLG. Accordingly, we characterized the heterostructure by micro-Raman spectroscopy using a visible laser tuned at $633 \mathrm{~nm}$. Figure 1(f) shows the Raman spectra at the SLG (red line) and tBLG (blue line) regions. The G-band peak at $1,584 \mathrm{~cm}^{-1}$, the $2 \mathrm{D}$ peak at $2,648 \mathrm{~cm}^{-1}$ and an additional peak at $1,621 \mathrm{~cm}^{-1}$ are observed for tBLG, while for SLG these bands are observed at $1,588 \mathrm{~cm}^{-1}(\mathrm{G})$ and $2,645 \mathrm{~cm}^{-1}$ (2D).

In the bilayer, the G-band peak has a two times higher intensity, as shown in the inset of Fig. 1(f). The enhancements of the G-band and the extra peak, $L_{a}$, testify the formation of a new band structure by interlayer coupling. They are thus an indication of the high crystalline quality of the samples and of a clean interface between the two monolayers forming the tBLG. The $\mathrm{L}_{\mathrm{a}}$ mode above the G-band position for the bilayer is activated by intralayer electron-phonon scattering from the unfolded longitudinal optical phonon (LO) [56] branch. The measured $L_{a}$ frequency of $1,621 \mathrm{~cm}^{-1}$ is consistent with the theoretical predictions [56] for a tBLG with $\theta \approx 7^{\circ}$. The LO dispersion is expected to have a non-monotonic dependence on the twist angle, with a maximum frequency at $\theta \approx 10^{\circ}$ but only slowly varying in the range of interest, thus making it unpractical to precisely determine the twist angle only from the Raman data.

We applied ultrafast TA microscopy to study the photophysics of tBLG samples as function of the twist angle. A NIR pump pulse with photon energy $E_{\text {pump }}$ is used to photo-excite the sample off-resonance with the vHs $\left(E_{\text {pump }}=0.800 \mathrm{eV}\right.$ for stack 1 and $E_{\text {pump }}=1.190 \mathrm{eV}$ for stacks $2-3$ ), while a second tunable pulse, the probe, monitors the relaxation of the induced hot carriers' distributions as function of pump-probe time delay $t$. Pump and probe beams are collinearly coupled to a confocal microscope to achieve $\mathrm{a} \approx 1 \mu \mathrm{m}$ spatial resolution. The differential transmission $(\Delta T / T)$ is measured as a function of the probe photon energy $E_{\text {probe }}$, using two distinct high sensitivity TA microscopes described in the Methods, guaranteeing a spectral coverage from 0.85 to $1.65 \mathrm{eV}$. Selected $\Delta T / T$ maps at fixed $E_{\text {probe }}$ and time delay $t=150 \mathrm{fs}$ are reported in Fig. 2, for three tBLG stacks with different $\theta$. As expected, the SLG regions exhibit a $\mathrm{PB}$ signal at all the investigated $E_{\text {probe, }}$ detected as a positive $\Delta T / T$ signal. The $\mathrm{PB}$ signal shows a smooth monotone increase towards lower energy, following the momentumspreading of the hot-carriers FD distribution.

This behavior contrasts to that of $\mathrm{tBLG}$, for which a complex dependence on $E_{\text {probe }}$ emerges shows also a PA signal. Specifically, under low photo-excitation fluences, we identified two characteristic components in the transient response: a clear peak in the $\mathrm{PB}$ and the appearance of PA band at lower energy. The PB peak corresponds to a factor 4-6 enhancement with respect to the SLG signal and it is centered in proximity to $E_{\mathrm{vHs}}$ (see first column of maps of Fig. 2). The PA is detected for $E_{\text {probe }}<E_{\mathrm{vHs}}$. At lower probe photon energies, about $300 \mathrm{meV}$ below $E_{\mathrm{vHs}}$, the $\Delta T / T$ signal of tBLG becomes barely distinguishable from that of SLG. While a similar behavior is observed in all the investigated samples, the energy position of $\mathrm{PB}$ and $\mathrm{PA}$ bands changes with $\theta$. The larger is the twist angle, the higher is $E_{\text {probe }}$ at which similar tBLG/SLG contrast is observed. In stacks 2 and 3, bilayer regions with different twist angle are present, so that the signal intensity evolves differently according to their orientation.

For all samples, the precise value of the twist angle is determined by recording the hot-PLE spectrum. The experimental
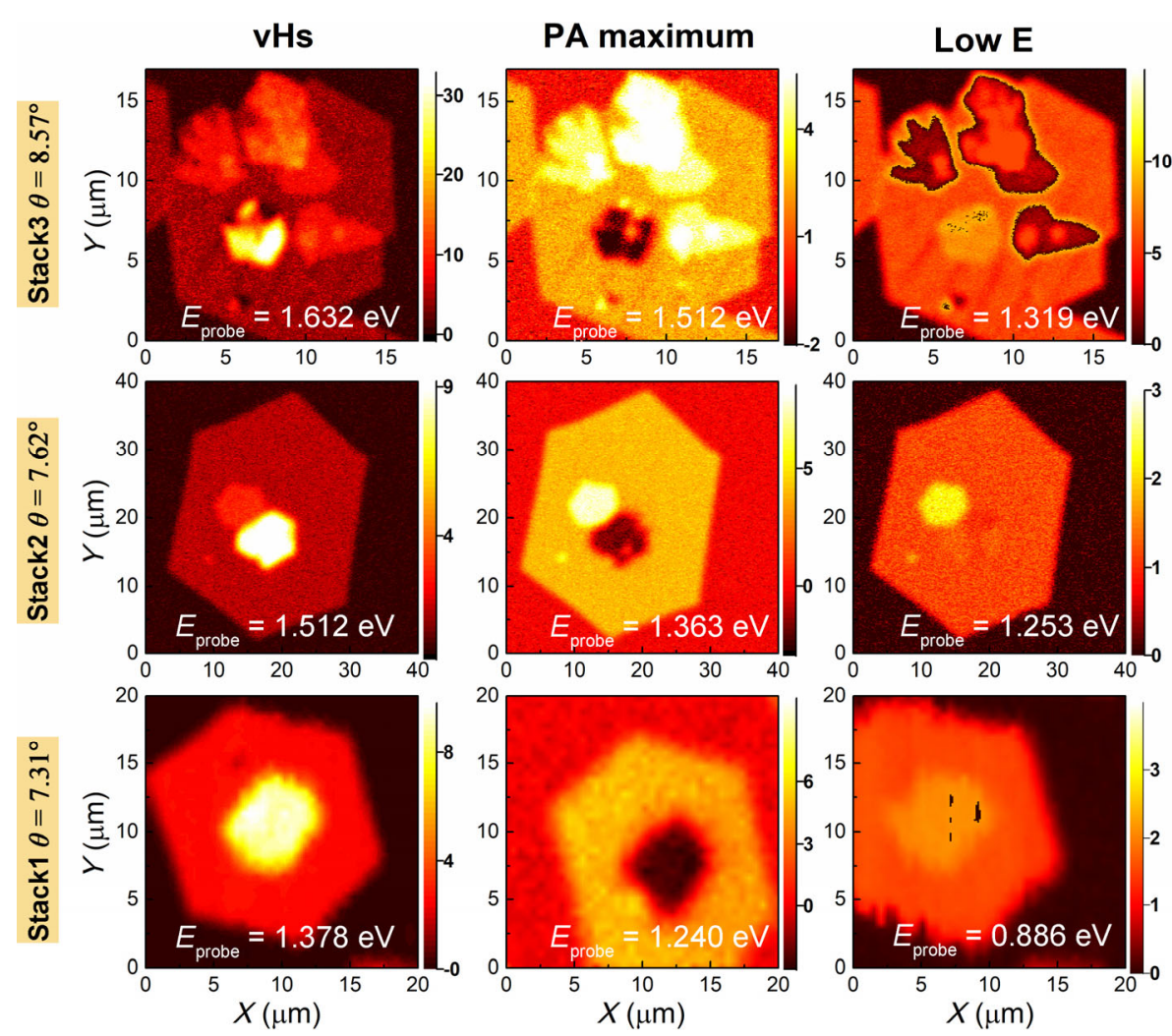

Figure 2 Color map images of the transient transmissivity $\Delta T / T\left(10^{-4}\right)$ at time delay $t=0$, for three tBLG stacks with twist angle increasing from the lower to the upper row of panels: $\theta=7.31^{\circ}, 7.62^{\circ}$, and $8.57^{\circ}$. The probe energies for the first column of maps are taken in resonance with the vHs at $E_{\text {probe }}=$ $1.632,1.512$, and $1.378 \mathrm{eV}$; for the second column at the maximum of the PA band at $E_{\text {probe }}=1.512,1.363$, and $1.240 \mathrm{eV}$; for the third column in resonance with the low-energy PB band at $E_{\text {probe }}=1.319,1.253$, and $0.886 \mathrm{eV}$, as indicated in the panels. 
$\Delta T / T$ spectra of monolayer and bilayer regions at $t=150 \mathrm{fs}$ for several tBLG samples are reported in Fig. 3(a), together with the PLE spectra used to extract $E_{\mathrm{vHs}}$ and $\theta$ (blue symbols).

As $\theta$ decreases, the overall TA spectrum experiences a red-shift. The PLE peak almost overlaps with the PB peak, with the larger mismatch appearing in the stack with the most intense PA band. The signal near the vHs, indeed, results from the interplay of $\mathrm{PA}$ and $\mathrm{PB}$ contributions whose relative intensity varies with the twist angle and excitation fluence. Remarkably, the PA emerges at low photo-excited carrier densities of the order of $n=1 \times 10^{13} \mathrm{~cm}^{-2}$, as estimated from an incident fluence of $50 \mu \mathrm{J} \cdot \mathrm{cm}^{-2}$ considering $4 \%$ absorption. At an incident fluence of $350 \mu \mathrm{J} \cdot \mathrm{cm}^{-2}$, corresponding to $n=7 \times 10^{13} \mathrm{~cm}^{-2}$, the PA band disappears and a PB is observed over all the spectral range (see Figs. S2 and S3 in the ESM). The lack of previous evidences $[47,48]$ of contribution from the ISB to the TA of tBLG could be traced back to the higher absorbed photon flux attained by pumping resonantly to the vHs. This dominance of the $\mathrm{PB}$ over the PA for high laser fluence was also observed for the intra- and inter-band transitions in suspended single layer graphene [31].

The dependence of the experimental TA spectrum on crystal orientation is traced back to the modifications of the phonon modes, electronic structure and of the JDOS, in which we theoretically compute as a function of the twist angle and the hot-electron temperature $T_{\mathrm{e}}$ (see details in the Methods section) [57]. Figure 3(b) shows the calculated JDOS considering the interband and intersubband transitions between electronic states for the initial hot carrier temperature of $1,500 \mathrm{~K}$ and same twist angles as in Fig. 3(a). The electronic states populated by the pump pulse allow for the ISB transitions. The temperature of the quasi-equilibrium hot electron distribution is obtained considering a three temperature model [58] (see Figs. S4 and S5 and details in the ESM). The black (red) curves in Fig. 3(b) show the JDOS for the interlayer ISB (interband-IB) transitions as a function of the twist angle $\theta$. The ISB curves are calculated for the electron and hole transitions as indicated in Fig. 1(c) by the solid and dashed red arrows, respectively. The higher energy peak in the JDOS for ISB transitions corresponds to holes' excitations, while the lower-energy peak to the electrons. The difference in energy for the electron and hole ISB transitions is due to the small difference of their curvatures. Note that, for all the twist angles, the electron ISB peak appears about $50 \mathrm{meV}$ below the IB peak due to the vHs transitions, while the hole ISB peak is almost overlapped with the vHs transitions (see Fig. S1 in the ESM). Figure 3(c) shows a comparison of both IB and ISB JDOS for carrier temperature of $1,500 \mathrm{~K}$ and room temperature $(300 \mathrm{~K})$; the blue arrows indicate that the ISB JDOS intensity increases with the temperature while the IB JDOS intensity decreases. The intensity of the JDOS is related to the dynamical conductivity (or absorption). In this way, the difference of the JDOS at these two temperatures is proportional to the $\Delta T / T$ spectrum and qualitatively explains the opposite sign of the contributions from ISB and IB transitions to the TA signal.

The increase of the JDOS associated to ISB transitions due to photo-excitation corresponds to the PA signal observed at energies just below $E_{\mathrm{vHs}}$ in the experimental data of Fig. 3(a). The ISB peak for holes is very close to the $E_{\mathrm{vHs}}$ transition that dominates the $\Delta T / T$ signal at these energies. The strong angle dependence of the PA energy position results in $\sim 250 \mathrm{meV}$ tuning with only a $\sim 1.26^{\circ}$ twist angle change. Model predictions of the PA energy position based on the JDOS calculation, with the folding method, agree very well with the experimental data. In the ESM, the expected values for a large range of twist angles (from $0^{\circ}$ to $30^{\circ}$ ) are reported in Fig. S1 in the ESM and appear always slightly red-shifted with respect to the vHs transition.

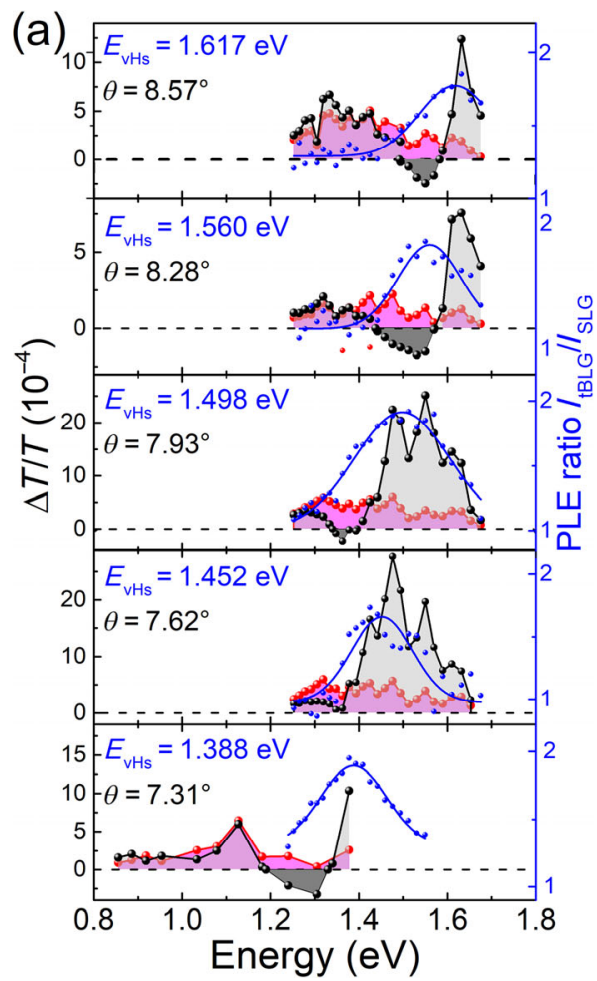

(b)

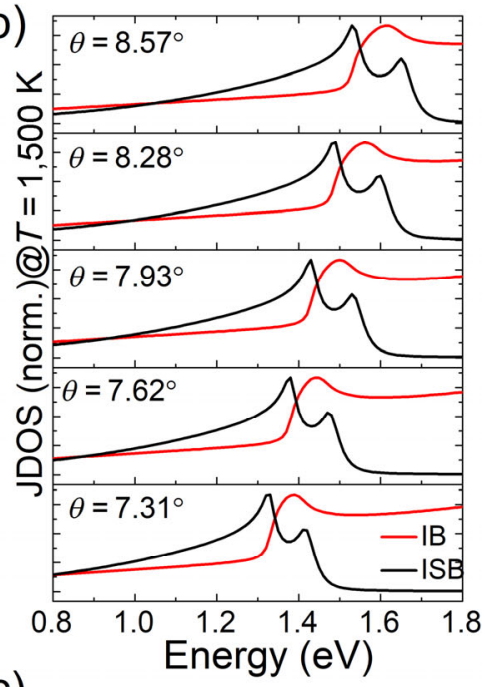

(c)

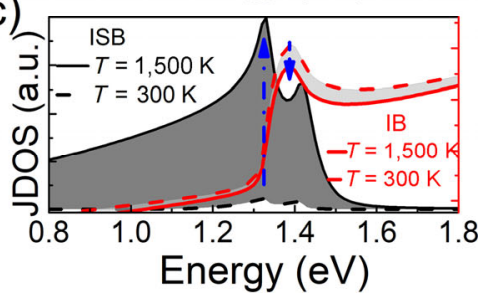

Figure 3 (a) Transient transmissivity spectra of SLG (red-dot and solid line) and tBLG (black-dot and solid line) at fixed time delay $t=150$ fs for samples with different $\theta$ as indicated by the labels. The blue dots are the PLE intensity ratio of the tBLG to the SLG fitted with a Gaussian function (blue solid line) peaked at $E_{\mathrm{vHs}}$ (b) Calculated JDOS for the ISB transition (black) and the $E_{\mathrm{vHs}}$ transition (red) for a hot electron temperature of 1,500 K. (c) A comparison of the IB and ISB JDOS for $1,500 \mathrm{~K}$ and the room temperature $300 \mathrm{~K}$. The difference of the JDOS at these two temperature is proportional to the $\Delta T / T$ spectrum. 
Figure 4 reports the $\Delta T / T$ dynamics of stack 1. A similar relaxation scenario is observed for all the stacks, once the shift of energy position of the transient spectrum is taken into account (see Fig. S6 in the ESM). The recovery dynamics of tBLG is consistently different from that of SLG. The dynamics of tBLG (full colored dots) is compared to that of SLG (open black symbols) for $E_{\text {probe }}$ corresponding to the main features of the optical response: the $\mathrm{PB}$ peak at the vHs, the PA corresponding to ISB transitions and the low-energy PB band. The $\Delta T / T$ dynamics in SLG is energy-independent and it is described by a bi-exponential decay convoluted to an instrumental response function (IRF) of $\approx 120 \mathrm{fs}$. The extracted time constants are $\tau_{1}{ }^{\text {SLG }}=120 \pm 20$ fs and $\tau_{2}{ }^{\text {SLG }}=1,980 \pm 60$ fs. These values are characteristic of SLG samples with low defect density [43]. The transient transmissivity depends superlinearly on $T_{\mathrm{e}}$ through the FD distribution function, so that the time evolution of $T_{\mathrm{e}}$ and $\Delta T / T$ are not correlated in time. However, while the fast relaxation component is strongly influenced by the superlinearity, the slow relaxation component of transient transmissivity is generally assumed as a reliable estimation of cooling time via anharmonic coupling of the thermalized bath of hot electrons and SCOPs with the acoustic phonons [59]. The $\Delta T / T(t)$ dynamics of tBLG, on the contrary, strongly depends on the transition energy even though, away from the $\mathrm{PA}$ and the vHs, it is very similar to that of SLG. At the vHs, the $\mathrm{PB}$ not only has a much higher initial intensity than for SLG, but it decays on a longer time scale. The dynamics, after deconvolution with the IRF, is best fit by a biexponential decay with $\tau_{1}{ }^{\mathrm{tBLG}}=140 \pm 20$ fs and $\tau_{2}{ }^{\mathrm{SLG}}=2,500 \pm 100$ fs. The PA band is best described by a three-exponential decay with time constants $\tau_{1}{ }^{\mathrm{tBLG}}=120 \pm 30 \mathrm{fs}, \tau_{2}{ }^{\mathrm{tBLG}}=1,960 \pm 40 \mathrm{fs}$ and $\tau_{3}{ }^{\mathrm{tBLG}}=$ $70 \pm 2$ ps with the additional decay component responsible for the tail extended to 100 ps in Fig. 4(b). Such long lifetimes can be explained by the peculiar temperature dependence of the JDOS that emerges at finite twist angles. We modeled the decay curves by the ratio of the calculated JDOS as a function of the electron temperature $T_{\mathrm{e}}$ (Figs. 4(c) and 4(d). We calculated the time evolution of $T_{\mathrm{e}}$ from a three temperature model, considering the electron energy relaxation through strongly coupled and weakly coupled phonon modes [58]. The specific heats for the electrons, SCOPs and weakly coupled phonons are obtained considering their density of states from tight-binding $[57,60]$ and force constant [61] methods parameterized by first principles calculations. For tBLG, it is important to include the additional low frequency and high density phonon modes at the $\Gamma$ point, that are not present in SLG, as representing the out-of-plane breathing of adjacent layers interacting via weak van der Waals forces (details in the ESM). The calculated $\Delta T / T(t)$ dynamics, both at the vHs transition and at the ISB transition, reproduces very well the experimental results. This indicates that the density of states enhancement at the vHs and the changes in the electron and phonon heat capacities can explain the observed long decay of the PA and PB bands.

To conclude, we demonstrated that at low excited carrier density the transient optical response of tBLG shows two peaks, one due to photoinduced absorption from intersubband transitions and another one due to photobleaching of the interband transitions in resonance with the $\mathrm{vHs}\left(E_{\mathrm{vHs}}\right)$. Similar to the vHs, the energy positions of these peaks can be controlled with the twist angle. At the vHs, we observed a carrier cooling time which is about $30 \%$ longer than for SLG, while the photoinduced intersubband signal shows a long-living tail up to 100 ps. A simple model, based on the JDOS enhancement and on the evolution of the electronic temperature as the hot electrons equilibrate with the tBLG phonon modes, explains well the experimental TA signal, both for the peaks position and for their temporal decays, without recurring to more complex multiparticle excitations as bound excitons or non-linear processes such as two-photon absorption.
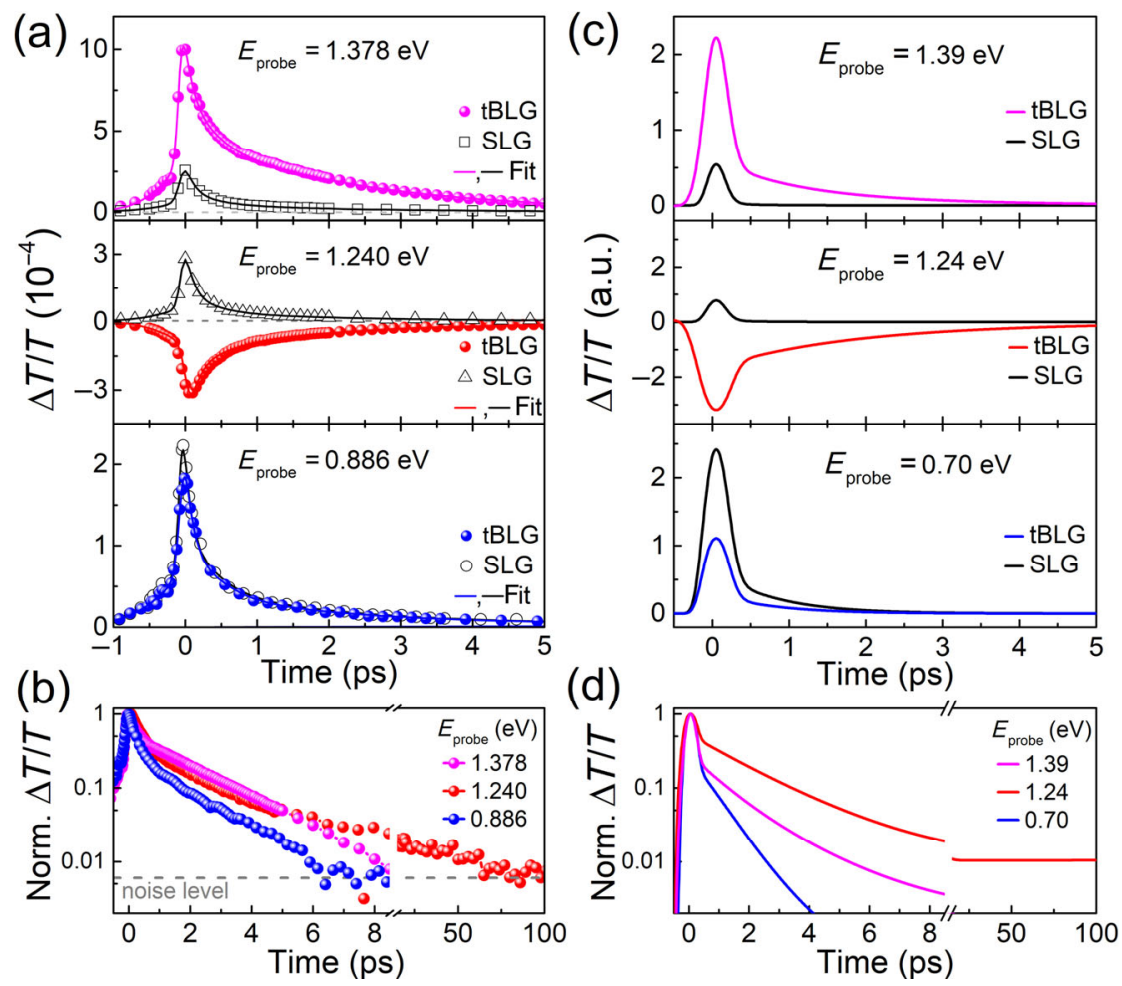

Figure 4 (a) $\Delta T / T$ dynamics of tBLG (full colored dots) and SLG (open black symbols) for the stack with $\theta=7.31^{\circ}$ at probe energy $E_{\text {probe }}=1.378 \mathrm{eV}$ corresponding to $E_{\mathrm{vHs}} ; 1.240 \mathrm{eV}$ corresponding to the ISB; $0.866 \mathrm{eV}$ in the low-energy PB band $E<E_{\mathrm{ISB}}$. (b) Comparison of $\Delta T / T(t)$ normalised to the maximum over an extended time scale. (c) Theoretically estimated decay curves based on the electron temperature time decay. In the simulations we used $E_{\text {probe }}=1.39,1.24$ and $0.70 \mathrm{eV}$ to best reproduce the experimental decay behavior. (d) Normalised theoretical curves obtained in (c). 


\section{Methods}

Sample preparation and characterization The twisted bilayer graphene single crystals were prepared by ambient pressure chemical vapor deposition (AP-CVD) synthesis on polycrystalline $\mathrm{Cu}$ foils. The nucleation conditions on $\mathrm{Cu}$ foils are controlled to allow both constituent layers to form as hexagonal single crystals, and each tBLG grain has a specific twist angle. The samples were transferred to a transparent amorphous quartz substrate for the optical images and spectroscopy measurements. More details about the sample preparation are presented in Ref. [62].

To select the samples for TA studies we first measured the sample twist angle by an analysis of the flake shapes on optical microscopy white light images. The optical images were obtained using an Olympus microscope (DP72) in reflection mode with a $100 \times$ objective lens. To obtain the twist angle we drew a hexagon for each layer and measured the angle between the edges of the two hexagons [52], as can be seen for the sample in Fig. 1(d).

We also characterized the sample twist angle by Raman spectroscopy using an alpha300R WITec confocal Raman spectrometer at excitation energy of $1.96 \mathrm{eV}(633 \mathrm{~nm})$. The Raman spectra were measured in backscattering geometry on a triple monochromator spectrometer equipped with a liquid-N2 CCD. All measurements were performed at room temperature.

For the PLE measurements a PL image was acquired for each excitation laser energy, that was tuned to cover the range of the vHs transition for each sample. The PL intensity was averaged at the bilayer region and divided by the PL at the monolayer region to obtain each point in the PLE spectra. The PL signal was measured in back scattering, separated from the excitation laser by a dichroic mirror (Semrock FF665-Di02). A bandpass filter $(75 \mathrm{~nm}$ centred at $610 \mathrm{~nm})$ was used in front of the detector to further block any scattered laser light (Chroma 610/75M).

High-sensitivity TA microscopy The TA data here reported have been acquired using two distinct microscopes working in complementary probe energy ranges, enabling an overall spectral coverage of $1 \mathrm{eV}$. The microscope used for the probe energy range of $0.8-1.4 \mathrm{eV}$ was based on a Er-doped fiber laser (Toptica-Femtofiber pro) generating $300 \mathrm{~mW}, 150 \mathrm{fs}$ pulses centered at $1,550 \mathrm{~nm}$ with $40 \mathrm{MHz}$ repetition rate. A portion of the output of the laser was used as the pump pulse tuned to $0.8 \mathrm{eV}$ and was modulated with an acousto-optic modulator operating at $1 \mathrm{MHz}$. The probe pulse extending from 0.73 to $1.45 \mathrm{eV}$ was obtained by super continuum (SC) generation from the laser fundamental focused in a highlynonlinear fiber. The low-energy branch of the SC and the fundamental were filtered out with a short pass filter cutting at $1,500 \mathrm{~nm}$. The pump and the probe beams were collinearly focused on the sample with an objective (Olympus-LCPLN-IR with magnification $100 \times$ and NA $=0.85$ ) to a spot size of about $1 \mu \mathrm{m}$. The probe transmitted by the sample was collected with an $8 \mathrm{~mm}$ focal length achromatic doublet, spectrally filtered with a monochromator with $5 \mathrm{~nm}$ resolution and detected by an InGaAs amplified photodiode with $4 \mathrm{MHz}$ bandwidth. The TA signal was measured with a lock-in amplifier with $300 \mathrm{~ms}$ effective time constant, resulting in a $\Delta T / T$ sensitivity of $10^{-7}$. TA dynamics was monitored by changing the pump-probe delay with an optical delay line, while the TA maps (images) at fixed probe photon energy and time delay were acquired by moving the sample with a motorized three-axis piezo-stack linear stage (Newport NPXYZ100).
The microscope used for the probe energy range of 1.25-1.65 eV was based on a commercial femtosecond optical parametric oscillator (OPO, Insight DS+, Newport, CA, USA) used as the laser source, with the fundamental 1,040 nm $(1.1923 \mathrm{eV})$ beam as the pump and the tunable output $750-990 \mathrm{~nm}(1.252-1.653 \mathrm{eV})$ as the probe. The pump beam was modulated at $20 \mathrm{MHz}$ by an electro-optical modulator, collinearly combined with the probe beam and delivered into a laser-scanning microscope (FV1200, Olympus). The laser beams were focused onto the sample with an air objective (UPLSAPO 20×, NA $=0.75$, Olympus) to about $1.5 \mu$ m diameter, raster scanned by a pair of galvo mirrors, transmitted through the sample, passed through an optical filter to block the pump beam, and then directed onto a large-area silicon photodiode. Pump fluence was kept at approximately $50 \mu \mathrm{J} \cdot \mathrm{cm}^{-2}\left(2 \times 10^{14}\right.$ incident photons $/ \mathrm{cm}^{2}$ ). The pump and probe beams were combined with a dichroic mirror (DMSP1000, Thorlabs) and aligned collinearly. The probe beam was optically filtered by two short-pass filters (FF011010/SP-25, Semrock), collected by a photodiode, and demodulated with a commercial lock-in amplifier (HF2LI, Zurich Instruments) to extract the TA signal. A pixel dwell time of $2 \mu$ s and image size of $320 \times 320$ pixels were used in the experiments. The cross-correlation between pump and probe pulses at the sample was measured to be $\sim 0.3 \mathrm{ps}$, and the time delay interval between adjacent images was set to $\Delta t=133$ fs. A single $t$ scan of 20 ps took about $1.5 \mathrm{~min}$, and a complete spectral scan took about $30 \mathrm{~min}$.

Theoretical model The electronic structures were obtained by folding the SLG calculation results. For SLG, we followed the procedure given in Refs. [57, 60], where the electronic structure calculations were based on a fifth neighbors tightbinding approach, with one orthonormalized $\mathrm{p}_{z}$ orbital per site, in which the hopping parameters were fitted to reproduce density functional theory (DFT) calculations with many-body corrections.

The electronic transitions between bands in tBLG were captured considering two SLG BZs twisted by a certain angle $\theta$. In this way, we considered only four electronic bands for the tBLG, one valence and one conduction band for each layer. For low temperatures, only the electronic IB transitions are possible. The magenta arrow in Fig. 1(c) shows the IB transition at the vHs, that gives rise to a peak in the JDOS at an energy $E_{\mathrm{vHs}}$ which is a function of $\theta$. When the temperature increases, ISB transitions between valence, or between conduction bands, are also possible (red arrows in Fig. 1(c)).

The allowed IB and ISB transitions do not depend on the size of the minigap, which appears due to the hybridization of the eigenstates near the band intersections. Our model cannot reproduce the interaction between layers and the atomic relaxation is not taken into account. However, Havener et al. [19] have shown that, although the interlayer interactions perturb the bands from each layer producing minigaps, which splits the valence and the conduction bands in $\mathrm{tBLG}$, the difference in energy between the minigaps is generally too small to be seen directly in the PL and absorption spectra, contributing only to the peak broadening. Also, since the allowed electronic transitions do not depend on the size of the minigap and the PL peak is usually symmetric, which means that both transitions from minigaps to the conduction and the valence bands contribute equally, the energy position of the JDOS peak obtained by the folding approach is supposed to give a good estimation of the twist angle. This justifies the folding approach used in our simulations, which does not consider interaction between layers $[19,56]$. 
In our model, it is possible to analyze the JDOS for IB and ISB transitions separately, as a function of the electronic temperature. We computed the JDOS as

$$
J\left(T_{\mathrm{e}}, E\right)=\frac{\gamma}{\pi N_{k}} \sum_{i, j, k} \frac{f\left(E_{i, k}, T_{\mathrm{e}}\right)-f\left(E_{j, k}, T_{\mathrm{e}}\right)}{\left(E_{i, k}-E_{j, k}+E\right)^{2}+\gamma^{2}}
$$

where the sum is performed on a uniform grid of $N_{k}=2,400 \times$ $2,400 \mathrm{k}$-points over the two dimensional $\mathrm{BZ}$ and for the different pairs of bands $i$; $j$ while $\gamma=1 \times 10^{-2} \mathrm{eV}$ is a small energy broadening parameter related to the inverse of the electron-hole lifetime. The function $f\left(E_{i, k}, T_{\mathrm{e}}\right)=\left[1+\exp \left(E_{i, k} / T_{\mathrm{e}}\right)\right]^{-1}$ is the Fermi function for a specific band energy $i$ of the state $\mathrm{k}$ and temperature $T_{\mathrm{e}}$ (the chemical potential $\mu$ is close to the Fermi level and both are set to zero).

Using only IB transitions in the JDOS calculation we found the energy peaks corresponding to the $E_{\mathrm{vHs}}$ for a specific twist angle $\theta$. For the same values of $E_{\mathrm{vHs}}$ obtained by the PLE peaks, we computed the normalized JDOS for IB and ISB transitions, as a function of the probe energy for $T_{\mathrm{e}}=1,500 \mathrm{~K}$, which is the estimated temperature for the hot electrons excited by the pump laser (see Figs. S4 and S5 in the ESM). We saw that the peaks corresponding to ISB transitions are always at lower energies than the IB transitions between the $\mathrm{vHs}$, as shown in Fig. 3(c).

An estimation of the $\Delta T / T$ dynamics can be obtained assuming that electron-radiation matrix element is constant for all transitions. In this way, the dynamical conductivity $\sigma$ (or absorption $\alpha$ ) becomes proportional to the JDOS which we computed including both the IB and the ISB as function of $T_{\mathrm{e}}$. Details of the $T_{\mathrm{e}}$ time-evolution calculation are described in the ESM.

\section{Acknowledgements}

We acknowledge financial support from Graphene FET Flagship Core 3 Project, Grant No. 881603 and the Brazilian funding agencies: FAPERJ (grant number E-26/010.101126/ 2018), Fapemig, CNPq, Capes and INCT Carbon Nanomaterials. Prof. Po-Wen Chiu is gratefully acknowledged for preparing the $\mathrm{tBLG}$ samples.

Funding note: Open Access funding provided by Politecnico di Milano.

Electronic Supplementary Material: Supplementary material (twist angle estimation from $E_{\mathrm{vHs}}$, fluence dependence of TA spectra, electron temperature calculation, and relaxation dynamics of tBLG stacks) is available in the online version of this article at https://doi.org/10.1007/s12274-021-3288-0.

Open Access: This article is licensed under a Creative Commons Attribution 4.0 International License, which permits use, sharing, adaptation, distribution and reproduction in any medium or format, as long as you give appropriate credit to the original author(s) and the source, provide a link to the Creative Commons licence, and indicate if changes were made.

The images or other third party material in this article are included in the article's Creative Commons licence, unless indicated otherwise in a credit line to the material. If material is not included in the article's Creative Commons licence and your intended use is not permitted by statutory regulation or exceeds the permitted use, you will need to obtain permission directly from the copyright holder.

To view a copy of this licence, visit http://creativecommons.org/licenses/by/4.0/.

\section{References}

[1] Geim, A. K.; Grigorieva, I. V. Van der Waals heterostructures. Nature 2013, 499, 419-425.

[2] Xia, F. N.; Wang, H.; Xiao, D.; Dubey, M.; Ramasubramaniam, A. Two-dimensional material nanophotonics. Nat. Photonics 2014, 8, 899-907.

[3] Ferrari, A. C.; Bonaccorso, F.; Fal'ko, V.; Novoselov, K. S.; Roche, S.; Bøggild, P.; Borini, S.; Koppens, F. K. L.; Palermo, V.; Pugno, N. et al. Science and technology roadmap for graphene, related two-dimensional crystals, and hybrid systems. Nanoscale 2015, 7, 4598-4810.

[4] Liu, Y.; Weiss, N. O.; Duan, X. D.; Cheng, H. C.; Huang, Y.; Duan, X. F. Van der Waals heterostructures and devices. Nat. Rev. Mater. 2016, $1,16042$.

[5] Novoselov, K. S.; Mishchenko, A.; Carvalho, A.; Neto, A. H. C. 2D materials and van der Waals heterostructures. Science 2016, 353, aac9439.

[6] Zhong, D.; Seyler, K. L.; Linpeng, X.; Cheng, R.; Sivadas, N.; Huang, B.; Schmidgall, E.; Taniguchi, T.; Watanabe, K.; McGuire, M. A. et al. Van der waals engineering of ferromagnetic semiconductor heterostructures for spin and valleytronics. Sci. Adv. 2017, 3, e1603113.

[7] Unuchek, D.; Ciarrocchi, A.; Avsar, A.; Watanabe, K.; Taniguchi, T.; Kis, A. Room-temperature electrical control of exciton flux in a van der Waals Heterostructure. Nature 2018, 560, 340-344.

[8] Gibertini, M.; Koperski, M.; Morpurgo, A. F.; Novoselov, K. S. Magnetic 2D materials and heterostructures. Nat. Nanotech. 2019, $14,408-419$

[9] Mishchenko, A.; Tu, J. S.; Cao, Y; Gorbachev, R. V.; Wallbank, J. R.; Greenaway, M. T.; Morozov, V. E.; Morozov, S. V.; Zhu, M. J.; Wong, S. L. et al. Twist-controlled resonant tunnelling in graphene/boron nitride/graphene heterostructures. Nat. Nanotechnol. 2014, 9, 808-813.

[10] Ribeiro-Palau, R.; Zhang, C. J.; Watanabe, K.; Taniguchi, T.; Hone, J.; Dean, C. R. Twistable electronics with dynamically rotatable heterostructures. Science 2018, 361, 690-693.

[11] Kockum, A. F.; Miranowicz, A.; De Liberato, S.; Savasta, S.; Nori, F. Ultrastrong coupling between light and matter. Nat. Rev. Phys. 2019, $1,19-40$.

[12] Dean, C.; Young, A. F.; Wang, L.; Meric, I.; Lee, G. H.; Watanabe, K.; Taniguchi, T.; Shepard, K.; Kim, P.; Hone, J. Graphene based heterostructures. Solid State Commun. 2012, 152, 1275-1282.

[13] Ju, L.; Shi, Z. W.; Nair, N.; Lv, Y. C.; Jin, C. H.; Velasco Jr, J.; Ojeda-Aristizabal, C.; Bechtel, H. A.; Martin, M. C.; Zettl, A. et al. Topological valley transport at bilayer graphene domain walls. Nature 2015, 520, 650-655.

[14] Cao, Y.; Fatemi, V.; Fang, S.; Watanabe, K.; Taniguchi, T.; Kaxiras, E.; Jarillo-Herrero, P. Unconventional superconductivity in magic-angle graphene superlattices. Nature 2018, 556, 43-50.

[15] Sunku, S. S.; Ni, G. X.; Jiang, B. Y.; Yoo, H.; Sternbach, A.; McLeod, A. S.; Stauber, T.; Xiong, L.; Taniguchi, T.; Watanabe, K. et al. Photonic crystals for nano-light in moiré graphene superlattices. Science 2018, 362, 1153-1156.

[16] Li, G. H.; Luican, A.; Dos Santos, J. M. B. L.; Neto, A. H. C.; Reina, A.; Kong, J.; Andrei, E. Y. Observation of van Hove singularities in twisted graphene layers. Nat. Phys. 2010, 6, 109-113.

[17] Zou, X. Q.; Shang, J. Z.; Leaw, J.; Luo, Z. Q.; Luo, L. Y.; La-O-Vorakiat, C.; Cheng, L.; Cheong, S. A.; Su, H. B.; Zhu, J. X. et al. Terahertz conductivity of twisted bilayer graphene. Phys. Rev. Lett. 2013, 110, 067401.

[18] Wang, Y. Y.; Ni, Z. H.; Liu, L.; Liu, Y. H.; Cong, C. X.; Yu, T.; Wang, X. J.; Shen, D. Z.; Shen, Z. X. Stacking-dependent optical conductivity of bilayer graphene. ACS Nano 2010, 4, 4074-4080.

[19] Havener, R. W.; Liang, Y. F.; Brown, L.; Yang, L.; Park, J. Van Hove singularities and excitonic effects in the optical conductivity of twisted bilayer graphene. Nano Lett. 2014, 14, 3353-3357.

[20] Alencar, T. V.; Von Dreifus, D.; Moreira, M. G. C.; Eliel, G. S. N.; Yeh, C. H.; Chiu, P. W.; Pimenta, M. A.; Malard, L. M.; de Paula, A. M. Twisted bilayer graphene photoluminescence emission peaks at van hove singularities. J. Phys. Condens. Matter 2018, 30, 175302.

[21] Dos Santos, J. M. B. L.; Peres, N. M. R.; Neto, A. H. C. Graphene bilayer with a twist: Electronic structure. Phys. Rev. Lett. 2007, 99, 256802 . 
[22] Tabert, C. J.; Nicol, E. J. Optical conductivity of twisted bilayer graphene. Phys. Rev. B 2013, 87, 121402.

[23] Moon, P.; Koshino, M. Optical absorption in twisted bilayer graphene. Phys. Rev. B 2013, 87, 205404.

[24] Rozhkov, A. V.; Sboychakov, A. O.; Rakhmanov, A. L.; Nori, F. Electronic properties of graphene-based bilayer systems. Phys. Rep. 2016, 648, 1-104.

[25] Yin, J. B.; Wang, H.; Peng, H.; Tan, Z. J.; Liao, L.; Lin, L.; Sun, X.; Koh, A. L.; Chen, Y. L.; Peng, H. L. et al. Selectively enhanced photocurrent generation in twisted bilayer graphene with van Hove singularity. Nat. Commun. 2016, 7, 10699.

[26] Sun, D.; Wu, Z. K.; Divin, C.; Li, X. B.; Berger, C.; De Heer, W. A.; First, P. N.; Norris, T. B. Ultrafast relaxation of excited dirac fermions in epitaxial graphene using optical differential transmission spectroscopy. Phys. Rev. Lett. 2008, 101, 157402.

[27] Newson, R. W.; Dean, J.; Schmidt, B.; Van Driel, H. M. Ultrafast carrier kinetics in exfoliated graphene and thin graphite films. Opt. Express 2009, 17, 2326-2333.

[28] Strait, J. H.; Wang, H. N.; Shivaraman, S.; Shields, V.; Spencer, M.; Rana, F. Very slow cooling dynamics of photoexcited carriers in graphene observed by optical-pump terahertz-probe spectroscopy. Nano Lett. 2011, 11, 4902-4906.

[29] Breusing, M.; Kuehn, S.; Winzer, T.; Malić, E.; Milde, F.; Severin, N.; Rabe, J. P.; Ropers, C.; Knorr, A.; Elsaesser, T. Ultrafast nonequilibrium carrier dynamics in a single graphene layer. Phys. Rev. B 2011, 83, 153410.

[30] Brida, D.; Tomadin, A.; Manzoni, C.; Kim, Y. J.; Lombardo, A.; Milana, S.; Nair, R. R.; Novoselov, K. S.; Ferrari, A. C.; Cerullo, G. et al. Ultrafast collinear scattering and carrier multiplication in graphene. Nat. Commun. 2013, 4, 1987.

[31] Malard, L. M.; Mak, K. F.; Neto, A. H. C.; Peres, N. M. R.; Heinz, T. F. Observation of intra- and inter-band transitions in the transient optical response of graphene. New J. Phys. 2013, 15, 015009.

[32] Song, J. C. W.; Rudner, M. S.; Marcus, C. M.; Levitov, L. S. Hot carrier transport and photocurrent response in graphene. Nano Lett. 2011, 11, 4688-4692.

[33] Graham, M. W.; Shi, S. F.; Wang, Z. H.; Ralph, D. C.; Park, J.; McEuen, P. L. Transient absorption and photocurrent microscopy show that hot electron supercollisions describe the rate-limiting relaxation step in graphene. Nano Lett. 2013, 13, 5497-5502.

[34] Gilbertson, S.; Dakovski, G. L.; Durakiewicz, T.; Zhu, J. X.; Dani, K. M.; Mohite, A. D.; Dattelbaum, A.; Rodriguez, G. Tracing ultrafast separation and coalescence of carrier distributions in graphene with time-resolved photoemission. J. Phys. Chem. Lett. 2012, 3, 64-68.

[35] Gierz, I.; Petersen, J. C.; Mitrano, M.; Cacho, C.; Turcu, I. C. E.; Springate, E.; Stöhr, A.; Köhler, A.; Starke, U.; Cavalleri, A. Snapshots of non-equilibrium Dirac carrier distributions in graphene. Nat. Mater. 2013, 12, 1119-1124.

[36] Ulstrup, S.; Johannsen, J. C.; Crepaldi, A.; Cilento, F.; Zacchigna, M.; Cacho, C.; Chapman, R. T.; Springate, E.; Fromm, F.; Raidel, C. Ultrafast electron dynamics in epitaxial graphene investigated with time-and angle-resolved photoemission spectroscopy. J. Phys. Condens. Matter 2015, 27, 164206.

[37] Song, J. C. W.; Reizer, M. Y.; Levitov, L. S. Disorder-assisted electronphonon scattering and cooling pathways in graphene. Phys. Rev. Lett. 2012, 109, 106602.

[38] Song, J. C. W.; Tielrooij, K. J.; Koppens, F. H. L.; Levitov, L. S. Photoexcited carrier dynamics and impact-excitation cascade in graphene. Phys. Rev. B 2013, 87, 155429.

[39] Betz, A. C.; Jhang, S. H.; Pallecchi, E.; Ferreira, R.; Fève, G.; Berroir, J. M.; Plaçais, B. Supercollision cooling in undoped graphene. Nat. Phys. 2013, 9, 109-112.

[40] Johannsen, J. C.; Ulstrup, S.; Cilento, F.; Crepaldi, A.; Zacchigna, M.; Cacho, C.; Turcu, I. C. E.; Springate, E.; Fromm, F.; Raidel, C. et al. Direct view of hot carrier dynamics in graphene. Phys. Rev. Lett. 2013, 111, 027403.

[41] Graham, M. W.; Shi, S. F.; Ralph, D. C.; Park, J.; McEuen, P. L. Photocurrent measurements of supercollision cooling in graphene. Nat. Phys. 2012, 9, 103-108.
[42] Tielrooij, K. J.; Song, J. C. W.; Jensen, S. A.; Centeno, A.; Pesquera, A.; Elorza, A. Z.; Bonn, M.; Levitov, L. S.; Koppens, F. H. L. Photoexcitation cascade and multiple hot-carrier generation in graphene. Nat. Phys. 2013, 9, 248-252.

[43] Alencar, T. V.; Silva, M. G.; Malard, L. M.; de Paula, A. M. Defectinduced supercollision cooling of photoexcited carriers in graphene. Nano Lett. 2014, 14, 5621-5624.

[44] Limmer, T.; Feldmann, J.; Da Como, E. Carrier lifetime in exfoliated few-layer graphene determined from intersubband optical transitions. Phys. Rev. Lett. 2013, 110, 217406.

[45] Ulstrup, S.; Johannsen, J. C.; Cilento, F.; Miwa, J. A.; Crepaldi, A.; Zacchigna, M.; Cacho, C.; Chapman, R.; Springate, E.; Mammadov, S. et al. Ultrafast dynamics of massive Dirac fermions in bilayer graphene. Phys. Rev. Lett. 2014, 112, 257401.

[46] Gierz, I.; Mitrano, M.; Petersen, J. C.; Cacho, C.; Turcu, I. C. E.; Springate, E.; Stöhr, A.; Köhler, A.; Starke, U.; Cavalleri, A. Population inversion in monolayer and bilayer graphene. J. Phys. Condens. Matter 2015, 27, 164204.

[47] Patel, H.; Havener, R. W.; Brown, L.; Liang, Y. F.; Yang, L.; Park, J.; Graham, M. W. Tunable optical excitations in twisted bilayer graphene form strongly bound excitons. Nano Lett. 2015, 15, 5932-5937.

[48] Patel, H.; Huang, L. J.; Kim, C. J.; Park, J.; Graham, M. W. Stacking angle-tunable photoluminescence from interlayer exciton states in twisted bilayer graphene. Nat. Commun. 2019, 10, 1445.

[49] Fukumoto, K.; Boutchich, M.; Arezki, H.; Sakurai, K.; Di Felice, D.; Dappe, Y. J.; Onda, K.; Koshihara, S. Y. Ultrafast electron dynamics in twisted graphene by femtosecond photoemission electron microscopy. Carbon 2017, 124, 49-56.

[50] Yang, H. Z.; Feng, X. B.; Wang, Q.; Huang, H.; Chen, W.; Wee, A. T. S.; Ji, W. Giant two-photon absorption in bilayer graphene. Nano Lett. 2011, 11, 2622-2627.

[51] Chu, Z. D.; He, W. Y.; He, L. Coexistence of van Hove singularities and superlattice Dirac points in a slightly twisted graphene bilayer. Phys. Rev. B 2013, 87, 155419.

[52] Ribeiro, H. B.; Sato, K.; Eliel, G. S. N.; De Souza, E. A. T.; Lu, C. C.; Chiu, P. W.; Saito, R.; Pimenta, M. A. Origin of van Hove singularities in twisted bilayer graphene. Carbon 2015, 90, 138-145.

[53] Havener, R. W.; Zhuang, H. L.; Brown, L.; Hennig, R. G.; Park, J. Angle-resolved Raman imaging of interlayer rotations and interactions in twisted bilayer graphene. Nano Lett. 2012, 12, 3162-3167.

[54] Latychevskaia, T.; Escher, C.; Fink, H. W. Moiré structures in twisted bilayer graphene studied by transmission electron microscopy. Ultramicroscopy 2019, 197, 46-52.

[55] Schmidt, H.; Rode, J. C.; Smirnov, D.; Haug, R. J. Superlattice structures in twisted bilayers of folded graphene. Nat. Commun. 2014, 5, 5742 .

[56] Eliel, G. S. N.; Moutinho, M. V. O.; Gadelha, A. C.; Righi, A.; Campos, L. C.; Ribeiro, H. B.; Chiu, P. W.; Watanabe, K.; Taniguchi, T.; Puech, P. et al. Intralayer and interlayer electron-phonon interactions in twisted graphene heterostructures. Nat. Commun. 2018, 9, 1221 .

[57] Vela, A.; Moutinho, M. V. O.; Culchac, F. J.; Venezuela, P.; Capaz, R. B. Electronic structure and optical properties of twisted multilayer graphene. Phys. Rev. B 2018, 98, 155135.

[58] Caruso, F.; Novko, D; Draxl, C. Photoemission signatures of nonequilibrium carrier dynamics from first principles. Phys. Rev. B 2020, 101, 035128.

[59] Tielrooij, K. J. Hesp, N. C.; Principi, A.; Lundeberg, M. B.; Pogna, E. A.; Banszerus, L.; Mics, Z.; Massicotte, M.; Schmidt, P.; Davydovskaya, D. et al. Out-of-plane heat transfer in van der Waals stacks through electron-hyperbolic phonon coupling. Nat. Nanotech. 2018, 13, 41-46.

[60] Venezuela, P.; Lazzeri, M; Mauri, F. Theory of double-resonant Raman spectra in graphene: Intensity and line shape of defectinduced and two-phonon bands. Phys. Rev. B 2011, 84, 035433.

[61] Cocemasov, A. I.; Nika, D. L.; Balandin, A. A. Phonons in twisted bilayer graphene. Phys. Rev. B 2013, 88, 035428.

[62] Lu, C. C.; Lin, Y. C.; Liu, Z.; Yeh, C. H.; Suenaga, K.; Chiu, P. W. Twisting bilayer graphene superlattices. ACS Nano 2013, 7, 2587-2594. 